\title{
Investigation of the optical properties of hollow aluminium 'nano-caps'
}

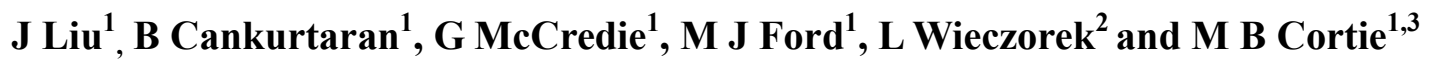 \\ ${ }^{1}$ Institute for Nanoscale Technology, University of Technology Sydney, PO Box 123, Broadway, NSW 2007, Australia \\ ${ }^{2}$ CSIRO Industrial Physics, PO Box 218, Lindfield, NSW 2070, Australia
}

E-mail : michael.cortie@uts.edu.au

\begin{abstract}
A colloidal suspension of hollow aluminium, cap-shaped nanoparticles ('nano-caps') can be conveniently produced by evaporation of aluminium onto a spin-coated layer of polystyrene nanoparticles (PSNPs), followed by sonication and dissolution of the polymer template. Although ordinary spherical aluminium nanoparticles have a plasmon resonance in the ultra-violet, the 'nano-caps' show plasmon absorption between 700 and $1200 \mathrm{~nm}$ due to their geometry. The position of their extinction peaks can be tuned by varying the thickness of the aluminium and the shape of the nano-cap. The optical properties of these shapes were modelled using the Discrete Dipole Approximation method, which confirmed that the 'caps' have very significantly red-shifted absorbance and scattering compared to spheres. This finding suggests that aluminium nano-caps might compete with gold and silver nanoparticles in applications requiring absorption in the near infrared.
\end{abstract}

PACS code : 52.25.Tx

\footnotetext{
${ }^{3}$ author to whom correspondence should be addressed
} 


\section{Aluminium 'nano-caps'}

\section{Introduction}

Nanoparticles of metal exhibit significantly different physical and chemical properties compared to the bulk elements ${ }^{[1]}$ and will in general exhibit a plasmon resonance with an appropriate wavelength in the electromagnetic spectrum. The resonance occurs at considerably lower frequencies than that of the bulk plasma frequency, $\omega_{\mathrm{p}}$, of the metal, which is an intrinsic material largely determined by its electron density. Particles with asymmetrical or more complex shapes such as rods, shells and prisms exhibit a plasmon resonance that is sensitively red-shifted even beyond that of solid spheres. Particle size, however, has only a modest influence on the position of the plasmon resonance. In the case of the noble metals $\mathrm{Au}$ or Ag, this permits the resonance to be shifted to the top of the visible, and even in some cases into the near-infrared ${ }^{[2-4]}$. This phenomenon has been extensively explored for its potential applications in subwavelength optics, data storage, light generation, microscopy, bio-photonics and immunochemistry ${ }^{[5]}$. Although the resonances of spherical nanoparticles have been known for over a century, and empirically exploited for far longer ${ }^{[6]}$, it has only been recently that methods to fabricate metal nanoparticles of the more complex shapes have been found. The study of protocols to fabricate or synthesize such particles is currently of great interest, because the particles can, for example, be potentially used in practical applications such as solar glazing coatings for windows ${ }^{[7]}$. The large red-shift of non-spherical or coreshell gold nanoparticles can also potentially be exploited for medical treatment of tumours ${ }^{[8]}$, while opal, inverse opal and mesoporous structures of silica, titanium and gold exhibit dramatic differences in optical properties at different incidence angles ${ }^{[9]}$.

Recently, the interesting prospects offered by the topologically related semi-shells ${ }^{\infty}$ have attracted interest. Half-shells ${ }^{[10]}$, nano-cups ${ }^{[11]}$ (somewhat misleadingly renamed recently as "nano-crescent moons ${ }^{,[12]}$, and nano-caps ${ }^{[13,14]}$ are members of this family (Figure 1), and all can be prepared by controlled physical or chemical deposition onto a template of some nanoparticle. When the semi-shells are arrayed on a surface in a fixed orientation they may make it superhydrophobic ${ }^{[10]}$, or they might confer

\footnotetext{
${ }^{\infty}$ semi-shell, partial shell, derived here from the prefix semi-, using its alternative English meaning of 'part' or 'partly', ie. a 'part-shell'.
} 


\section{Aluminium 'nano-caps'}

optical properties that depend on the angle of inclination of light or measurement ${ }^{[11,14]}$. Polymer nanoparticles offer a convenient template for such investigations and have been extensively exploited for the fabrication of a variety of nanoparticles having creative shapes, with various properties and potential uses $^{[15-18]}$.

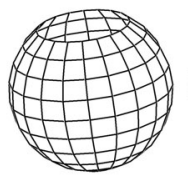

a

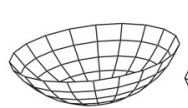

e

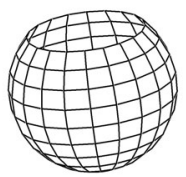

b

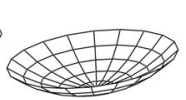

f

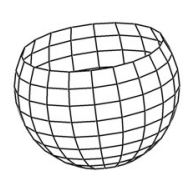

C

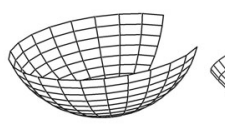

g

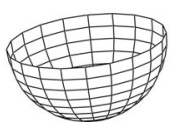

d

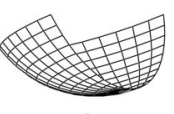

$\mathrm{h}$

Figure 1. The range of forms taken up by semi-shells, a) to c) nano-cups, d) a half-shell, e) and f) nanocaps with rotational symmetry, g) and h) nano-caps of low symmetry.

We have recently successfully prepared suspensions of discrete, hollow nano-caps of gold and other metals, including aluminium, using templates of sacrificial polymer nanoparticles, and these shapes were found to have a significantly red shifted optical absorption ${ }^{[13]}$. The key to producing separatable nano-caps is to control the angle of deposition of the metal onto the template so that the caps are not welded together. However, while the chemical inertness and other special properties of gold make it particularly useful in niche areas, its high cost impedes commercialization. Therefore methods to achieve similar optical properties with nanoparticles of cheaper metals could be of great value. In the present work we specifically address the properties of aluminium nano-caps, and in particular examine the effect of deposition thickness on their optical properties. In fact, the plasmon absorption and scattering of light by spherical aluminium nanoparticles has already been well studied ${ }^{[19]}$; the thin alumina films invariably associated with aluminium exhibit infrared absorption ${ }^{[20]}$ while the plasmon excitations of the nanospheres and nano-shells have been investigated both experimentally ${ }^{[21]}$ and theoretically ${ }^{[22]}$. However, there appears to have been little work on aluminium nanoparticles of non-spherical shape. In this paper 


\section{Aluminium 'nano-caps'}

we confirm the large red-shift in the plasmon resonance of nano-caps of aluminium, and suggest that this invites potential applications in the NIR region. Calculations were carried out to examine the influence of the cap shape on the optical properties, and to clarify the relative contributions of absorption and scattering to the extinction spectra.

\section{Experimental}

The fabrication of the nano-caps began with the application of commercial $200 \mathrm{~nm}$ polystyrene nanoparticles (PSNP) from Spherotech Inc onto a flat glass substrate by spin coating. The PSNP solution for the spin coating was prepared by mixing $10 \mu \mathrm{l}$ PSNP (5\% by volume) with $120 \mu 1$ of a $1: 400 \mathrm{v} / \mathrm{v}$ mixture of Triton X-100 and methanol. Deposition of the coating was carried out using a spin coater (Headway Research Inc.) at $3600 \mathrm{rpm}$ in 20 seconds. Thereafter the sample was placed in the evaporation apparatus and the chamber pressure was reduced to less than $\sim 3 \times 10^{-4}$ Pa prior to the deposition of a layer of aluminium. A quartz crystal micro-balance for monitoring of thickness was calibrated by applying the parameters of density $\left(2.7 \mathrm{~g} . \mathrm{cm}^{-3}\right)$ and acoustic impedance $\left(8.17 \mathrm{~g} . \mathrm{cm}^{-2} \mathrm{sec}\right)$ for aluminium. However, the thickness of aluminium deposited on the spheres will obviously vary from a maximum for surfaces inclined at right angles to the flux of metal, to zero for those parallel to it. The aluminium flux was at $50^{\circ}$ to the normal of the PSNP-coated sample substrate. Next, the separation of the aluminium nano-caps was carried out in clean dichloromethane under sonication (Cell Disruptor, Heat Systems-Ultrasonics Inc) for 5 min. The sonicated mixture was then centrifuged to remove most of the PSNP, and purified aluminium nano-caps were obtained after two cycles of washing and centrifuging using a wash of clean $\mathrm{CH}_{2} \mathrm{Cl}_{2}$ and Milli Q water. The remaining residue of large plates of aluminium and any agglomerated nano-caps were readily separated from the discrete particles by ordinary gravity settling of the re-suspended mixture. All the SEM images reported in this paper were taken in a LEO-SEM (Supra 55VP, Zeiss) using an in-lens detector. The optical transmission spectra of particles suspended in $\mathrm{CH}_{2} \mathrm{Cl}_{2}$ were taken in a Carey 5E UVvis-NIR spectrometer. 


\section{Aluminium 'nano-caps'}

The Discrete Dipole Approximation (DDA ${ }^{[23]}$ method was used to theoretically model the optical response of the nano-caps in order to identify the source of peaks observed in the experimentally determined extinction spectra. The DDA method calculates the total cross sections for absorption and scattering for an arbitrarily shaped target. We used the DDSCAT 6.1 implementation of Bruce T. Draine ${ }^{[23]}$. DDSCAT uses an array of polarizable points to approximate the target geometry, however the geometry of the present nano-caps was not available in the standard DDSCAT implementation. Therefore it had to be defined and imported into DDSCAT. By borrowing from the realm of computer graphics techniques we opted to implement a computer program which generated the nano-cap geometry using a simplified ray tracing algorithm ${ }^{[24]}$. Ray tracing was chosen because it closely resembles the physical process of evaporating aluminium particles onto the polystyrene spheres, and can handle shadowing of one sphere by another. Instead of tracing light rays from the camera to light sources, aluminium particles were traced from locations on the polystyrene spheres to the aluminium particle emitter.

The computational time required for calculating the absorption and scattering cross sections for these complex geometries is quite significant. With cpu times of approximately 150 to 250 hours (depending on the target and the chosen accuracy of the results) we opted to run the calculations on a cluster. The long computational times are due to the number of dipoles required to accurately describe the targets and predominately due to the difficulty of the DDA method to converge to a correct result when working with the refractive index of metals (i.e. when $|m|>2$, where $m$ is the complex refractive index, the DDA method converges very slowly ${ }^{[23]}$ ). The number of dipoles used here for each target well exceeded any criterion DDSCAT required for describing arbitrarily shaped targets.

\section{Results and Discussion}

\subsection{Experimental results}

Micrographs of aluminium nano-caps before and after separation from the PSNPs are shown in Figure 2. The aluminium grains (crystallites) can be clearly observed in Figure 2(a) and 2(b). The grain size increased when thicker coatings of aluminium were deposited, consistent with the observation of 


\section{Aluminium 'nano-caps'}

Hoffmann et al. ${ }^{[25]}$. When the suspension of the aluminium nano-caps in $\mathrm{CH}_{2} \mathrm{Cl}_{2}$ was settled for $24 \mathrm{~h}$, the nano-caps would agglomerate and precipitate out (Figure 2(c)). Images of single aluminium nano-caps of different shapes are shown in Figure 2(d) to (f). Analysis of these shapes shows that the form taken by the nano-caps depends not only upon the orientation of the plane of PSNPs with respect to the Al source, but also on the rotation of the PSNP structure within its own plane. If for example the hexagonal arrangement of PSNPs is assigned crystallographic coordinates as if it were a basal plane, then evaporation along, for example, $\langle\mathbf{1 0 1 1}\rangle$ produces a different form of nano-cap than along $\langle 2 \overline{1} \overline{1} 1\rangle$ due to a difference in shadowing geometry. Interestingly some nano-caps were found to join together to form dimers (Figure $2(\mathrm{~g})$ and $(\mathrm{h}))$. The nano-caps were confirmed to be pure aluminium by energy dispersive X-ray

spectroscopy.

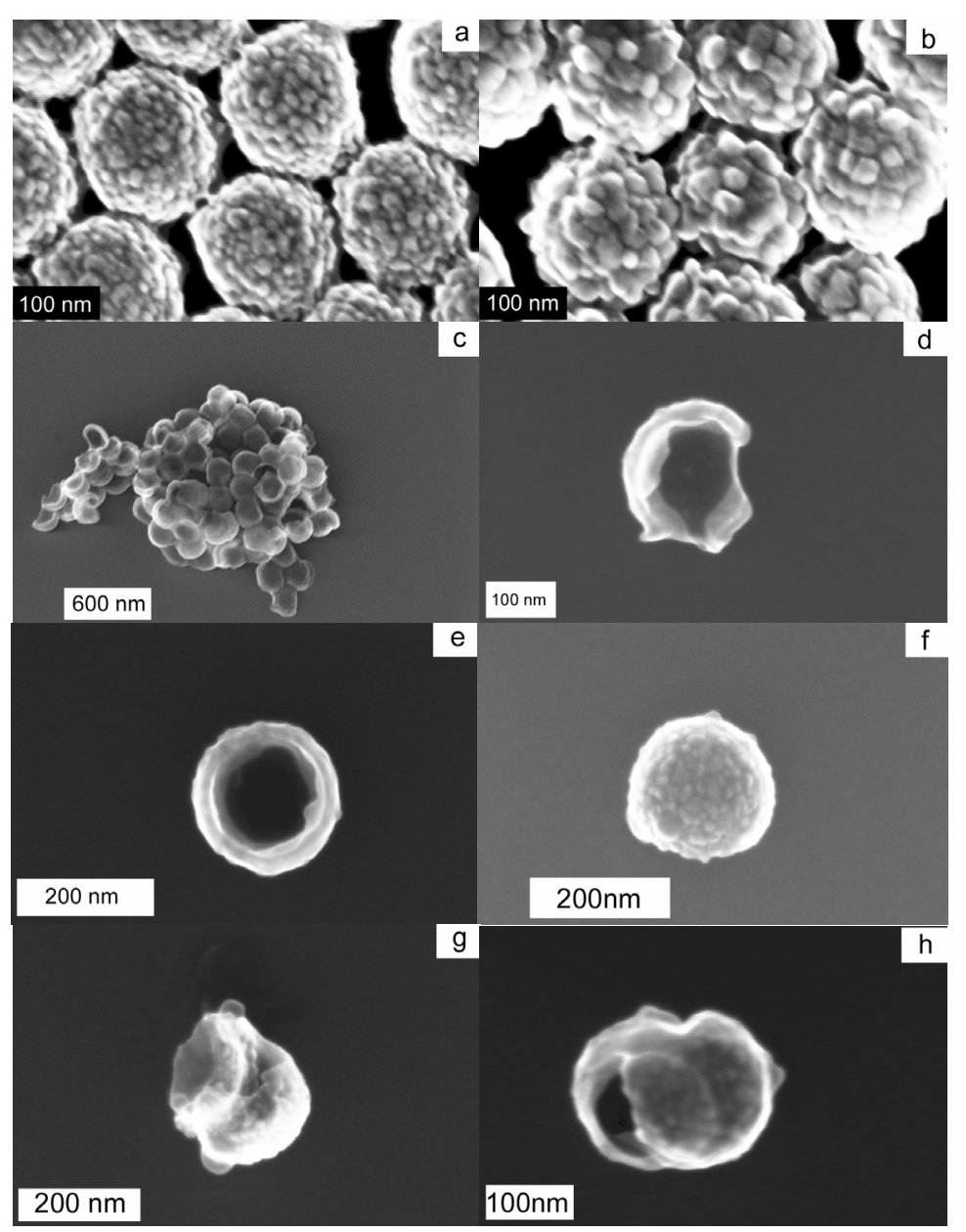




\section{Aluminium 'nano-caps'}

Figure 2. Micrographs of aluminium nano-caps, a) $14 \mathrm{~nm}$ aluminium deposit before peeling off from the PSNPs, b) $26 \mathrm{~nm}$ deposit before peeling off from the PSNPs, c) the aggregated nano-caps after separation from PSNPs, d) - f) single nano-caps of different shapes, g), h) nano-cap dimers.

The optical extinction spectra of a series of nano-caps suspended in clean $\mathrm{CH}_{2} \mathrm{Cl}_{2}$ and of different nominal deposit thicknesses are shown in Figure 3(a). The peaks in the transmission spectra at $\sim 1000 \mathrm{~nm}$ are due to a combination of the dipole plasmon resonance and scattering of single nano-caps. Another small peak (indicated as a plateau) at $790 \mathrm{~nm}$, which might be due to a quadrupole resonance ${ }^{[11]}$ was also observed. The peak positions of the extinction at $\sim 1000 \mathrm{~nm}$ were examined and it was found that the they were slightly blue-shifted with an increase of the aluminium thickness (Figure 3(b)). This phenomenon is consistent with what we observed previously with gold nano-caps ${ }^{[13]}$. Since the nano-caps are randomly suspended in the $\mathrm{CH}_{2} \mathrm{Cl}_{2}$, they will have all the possible orientations to the incidence light, which causes some of the peak broadening ${ }^{[13]}$. In addition, another reason for broadening of the peaks might be the formation of dimer, trimer or higher aggregations of suspended particles ${ }^{[26]}$. We have taken care to exclude any residual contribution of the polymer, solvent or cuvette to these spectra and confirmed that these absorptive phenomena are only caused by the nano-caps. 


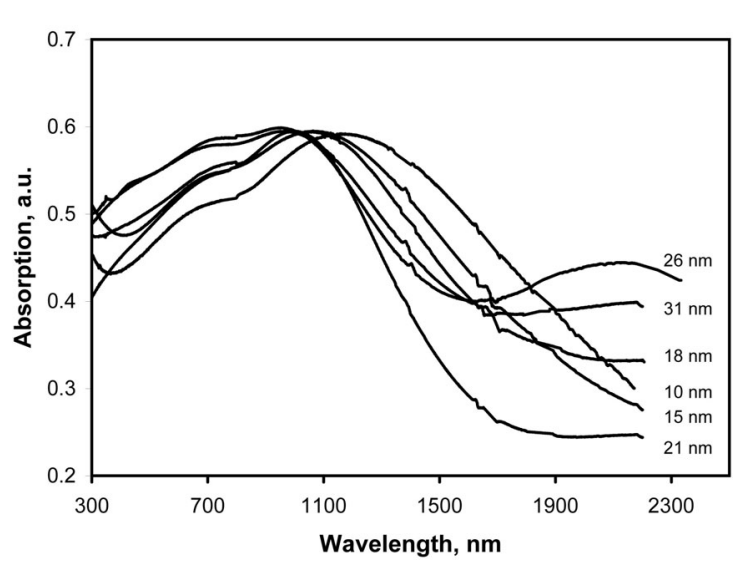

(a)

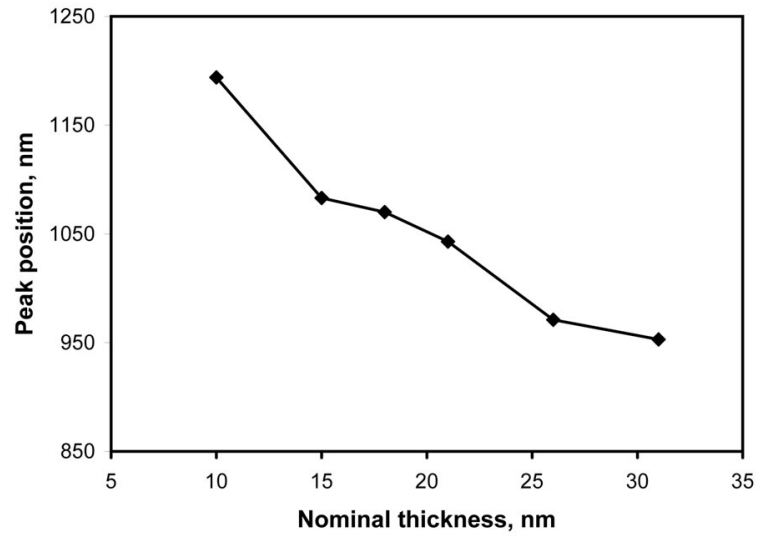

(b)

Figure 3. Effect of thickness of nano-cap on optical extinction, a) optical extinction of nano-caps deposited at $50^{\circ}$ for a range of nominal deposition thicknesses, b) position of major extinction peak as a function of deposit thickness.

An interesting aspect visible in Figure 3(a) is the absorptive peak at $2200 \mathrm{~nm}$, which is most clearly evident when the nominal thickness of the deposited aluminium was controlled at $26 \mathrm{~nm}$. Both increase and decrease of the thickness from that value reduced the magnitude of this peak. Therefore we believe that this presence of this peak must be closely related to the cap thickness. In the case of true nanoshells the ratio of the core diameter to the shell thickness would have to be more than 40 to produce a plasmon resonance at $1900 \mathrm{~nm}$. With our caps the ratio of diameter to thickness will not reach such a high value. Therefore we suggest that this peak in the present nano-caps at $2200 \mathrm{~nm}$ should rather be attributed to a multipolar resonance resulting from the reduced symmetry of these particles.

Theoretical calculations of the optical properties of ordinary Al nano-spheres indicate that they have plasmon excitations in the UV region ${ }^{[13]}$. However, the presence of surface oxide, deviations from spherical geometry, and scattering phenomena are known to cause significant red-shifting of their extinction spectra $^{[19]}$. The question therefore is whether extinction spectra like those in Figure 3 could have been obtained from ordinary aluminium nanoparticles. For this reason, we also examined the spectra of suspensions of gas atomized aluminium spheres of different sizes in $\mathrm{CH}_{2} \mathrm{Cl}_{2}$ (Figure 4). SEM examination of these powders indicated that they were not monodisperse, and not perfectly spherical. The 


\begin{abstract}
Aluminium 'nano-caps'
strongest extinction for the smallest nanospheres ( $\sim 35 \mathrm{~nm}$ diameter) was centred at $509 \mathrm{~nm}$ (Figure 4(a)). Similar extinction characteristics were observed for nanospheres with larger size ( $\sim 60 \mathrm{~nm}$, Figure 4(b)). However when the size of the aluminium spheres becomes larger still $(\sim 120 \mathrm{~nm})$, the spectra showed broad absorption across the NIR region (Figure 4(c)). Of course none of these diameters exactly matches those of the nanocaps, but we contend that a comparison of Figures 3 and 4 shows that the extinction spectra of these nominally spherical and solid aluminium powders are quite different in nature from those of the aluminium nano-caps.
\end{abstract}


Aluminium 'nano-caps'

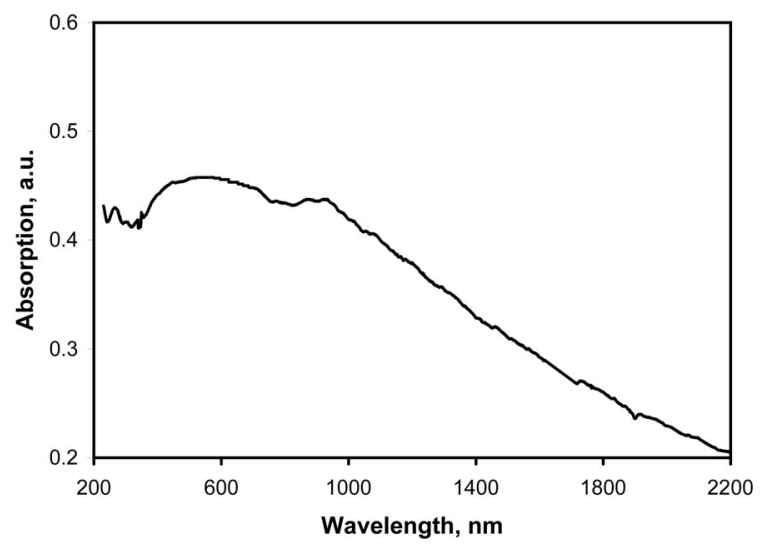

(a)

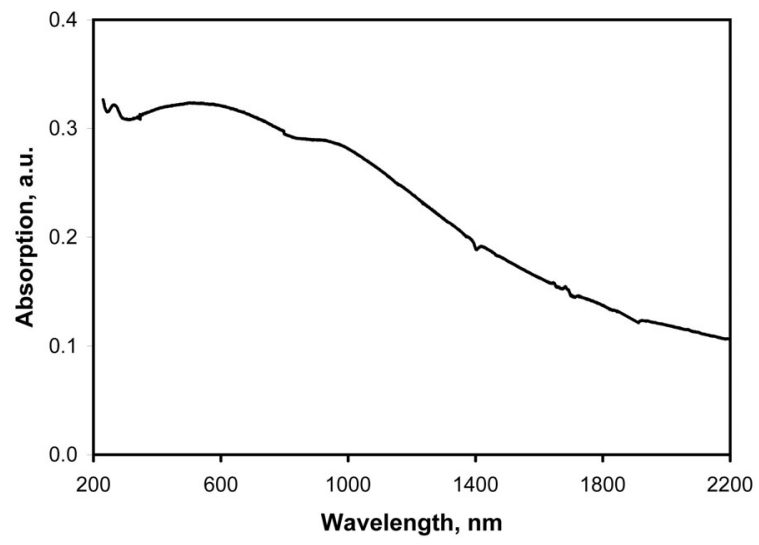

(b)

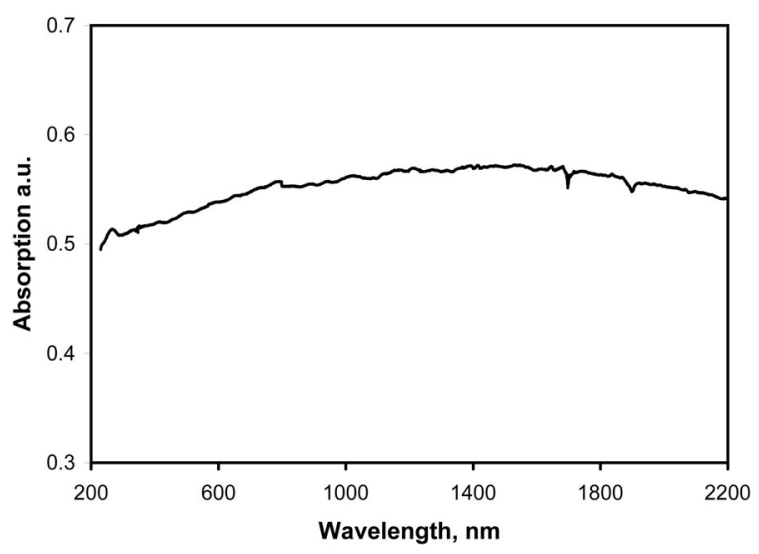

(c)

Figure 4. The optical extinction spectra of aluminium nanospheres of different sizes. (a) $\sim 35 \mathrm{~nm}$ diameter. (b) $\sim 60 \mathrm{~nm}$ diameter and (c) $\sim 120 \mathrm{~nm}$ diameter. 


\section{Aluminium 'nano-caps'}

\subsection{Computational results}

Figure 5(a) shows a surface covered with polystyrene spheres from the viewpoint of a particle emitter at an inclination of $50^{\circ}$ to it. The visible regions will be coated with metal, the obscured regions will not. It is easily seen that any shadows cast by adjacent spheres define the shape of the nano-caps. Figures $5(\mathrm{~b})$ to (d) show the actual target geometries generated using ray tracing. Figure 5(b) represents a $200 \mathrm{~nm}$ wide quarter shell, Figure 5(c) represents a $200 \mathrm{~nm}$ quarter shell with a large portion missing and two sharp prongs, and Figure 5(d) represents an $180 \mathrm{~nm}$ quarter shell with two portions missing and three sharp prongs. However, unlike the real nano-caps, which are expected to have a variable thickness, these computer-generated targets had a uniform shell thickness. These three geometries were chosen due to the fact that they are the most commonly found amongst all possible target geometries created using the evaporation technique.
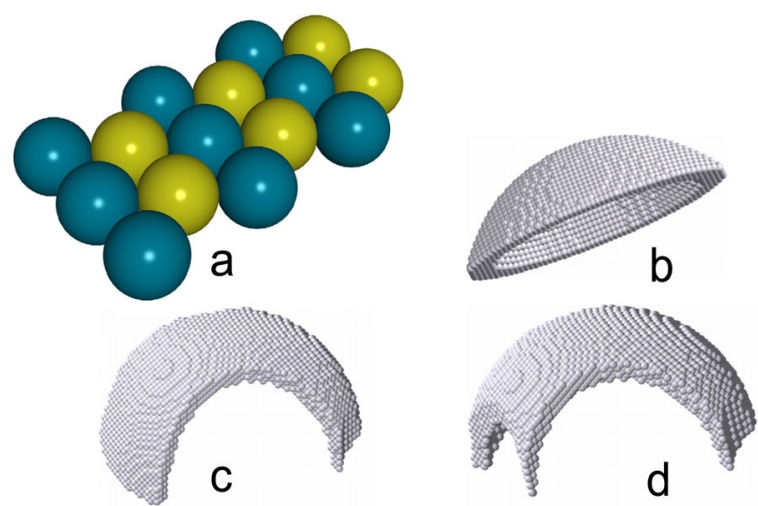

Figure 5. Target geometries used in the DDSCAT calculations, a) ray traced image of the polystyrene sphere lattice as seen from the particle emitter, b), c), d) common geometries created using the evaporation technique. The number of dipoles in b), c) and d) is 11272,14774 and 12800 respectively.

The calculated efficiency factors for optical extinction of the three target geometries are shown in Figure 6 for a shell thickness of $26 \mathrm{~nm}$. The calculated efficiency factor for a complete nano-shell of thickness $26 \mathrm{~nm}$ and diameter $200 \mathrm{~nm}$ is also plotted for purposes of comparison. All four spectra are 


\section{Aluminium 'nano-caps'}

relatively broad and exhibit a number of peaks. The efficiency factors shown in Figure 6 are the sum of the individual efficiency factors due to scattering and absorption, Figure 7. While the overall efficiency does not show any remarkable differences among the four shapes, details of the scattering and absorption vary quite significantly. The proportion of absorption to scattering increases quite significantly from the complete shell to the quarter shell, two-prong and finally three-prong shell. For both the two-and threeprong shells, the extinction is predominantly due to absorption.

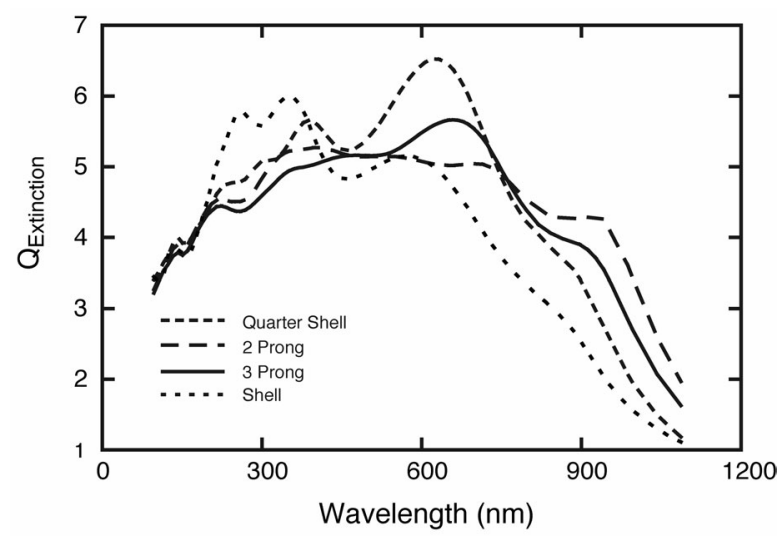

Figure 6. Orientation averaged extinction efficiency for target shapes quarter, two prong and three prongs.

The calculated absorption spectrum for the complete shell shows peaks at approximately 250,320 and $600 \mathrm{~nm}$. Within the framework of Mie theory one would expect ${ }^{[27]}$ at least the first and second order plasmon resonances to contribute to the absorption spectrum of a solid spherical particle of $200 \mathrm{~nm}$ diameter. Prodan et al. ${ }^{[28]}$ have extended this treatment to nanoshells and shown that the absorption spectrum can be considered as equivalent to a sum of plasmon resonances on the surface of a solid sphere and inside a cavity of the appropriate radius. As the shell gets thinner these two resonances interact more strongly and their energies shift away from the isolated sphere and cavity resonances; they are hybridized. The interaction is expected to be quite strong for the nanoshells shown in Figure 6. According to the formula of Prodan et al., the hybridized $l=1$ and $l=2$ resonances for the complete shell should occur at approximately $185 \mathrm{~nm}, 190 \mathrm{~nm}, 440 \mathrm{~nm}$ and $570 \mathrm{~nm}$. These calculations assume the bulk plasmon resonance in $\mathrm{Al}$ is at $15 \mathrm{eV}$. Higher-order resonances are predicted to occur at wavelengths between 185 


\section{Aluminium 'nano-caps'}

$\mathrm{nm}$ and $570 \mathrm{~nm}$. However, it is difficult to draw a convincing correspondence between the calculated absorption efficiency and the predicted plasmon resonance wavelengths for the complete shell. Interpretation of the absorption efficiencies for the other three shapes is complicated further by their reduced symmetry. From Figure 7 it would appear that this loss of symmetry increases the absorption efficiency at longer wavelengths, particularly in the 600 to $1000 \mathrm{~nm}$ range compared with absorption in the complete shell, and that the introduction of sharp prongs into the shape gives particularly efficient absorption over this wavelength range.

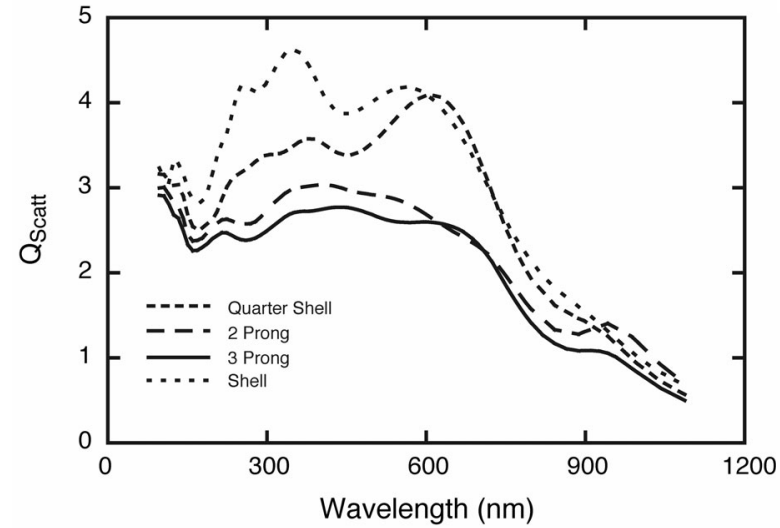

(a)

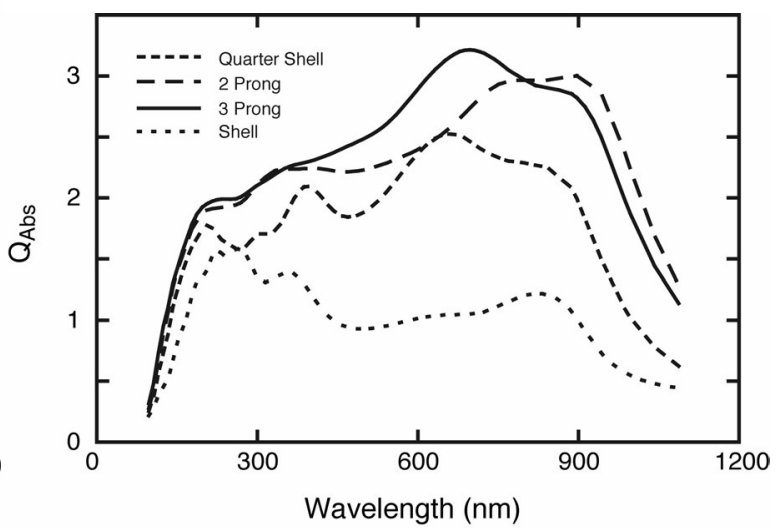

(b)

Figure 7. Orientation averaged efficiency factors, a) scattering, b) absorption.

Figure 8 shows a normalized comparison between the experimental absorption data and the total extinction efficiency calculated for the two- and three-prong targets. It is apparent that the general shape of the experimental extinction spectra is reasonably well represented by the calculated absorption efficiencies. The residual discrepancy between the calculated and measured spectra is likely to be due to the fact that the real caps had a variable thickness, a grainy surface morphology and a distribution of shapes. The grainy morphology in particular has recently been shown to red-shift the extinction spectra of shells ${ }^{[29]}$ and an analogous effect could be expected for the caps. 


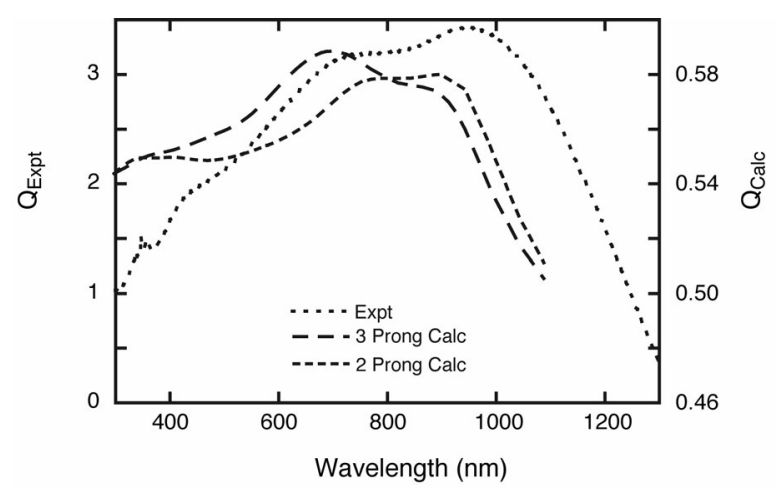

Figure 8. Comparison plot between experimentally found absorption spectrum of a suspension of 200nm diameter aluminium nano-caps with nominal $26 \mathrm{~nm}$ thickness, and the spectra calculated for two- and three-pronged nano-caps.

\section{Conclusions}

Discrete nano-caps of aluminium were fabricated by a process involving spin-coating of polystyrene nanoparticles as a template, followed by deposition of aluminium of different thicknesses by evaporation at an angle of $50^{\circ}$, and then finally sonication in $\mathrm{CH}_{2} \mathrm{Cl}_{2}$. The resulting suspensions of the nano-caps were stable and exhibited enhanced optical extinction centred at $1070 \mathrm{~nm}$ in the NIR region. A second peak in optical extinction was obtained at $2200 \mathrm{~nm}$ with the nano-caps of a nominal $26 \mathrm{~nm}$ in thickness. The optical properties of nano-caps of defined physical geometries were calculated using the Discrete Dipole Approximation. The simulated spectra gave an adequate qualitative representation of the measured ones, with the residual discrepancies between the two being evidently due to imperfections in the form of the real caps. The extinction spectrum of the quarter shell is evidently dominated by scattering, while that of the three pronged nano-cap has a strongly absorptive component.

\section{Acknowledgments}

The authors thank CSIRO, Australia, and the University of Technology Sydney. 


\section{Aluminium 'nano-caps'}

\section{References}

[1] Eberhardt W 2002 Surf. Sci. 500242.

[2] Kelly K L, Coronado E, Zhao L L and Schatz G C 2003 J. Phys. Chem. B 107668.

[3] Oldenburg S J, Jackson J B, Westcott S L and Halas N J 1999 Appl. Phys. Lett. 752897.

[4] Liz-Marzan, L M 2004 Mater. Today 7(2) 26.

[5] Xia Y and Halas N 2005 MRS Bulletin 30338.

[6] Wagner F E, Haslbeck S, Stievano L, Calogero S, Pankhurst Q A and Martinek P 2000 Nature 407 691.

[7] Xu X D, Stevens M and Cortie M B 2004 Chem. Mater. 162259.

[8] O'Neal D P, Hirsch L R, Halas N J, Payne J D and West J L 2004 Cancer Lett. 209171.

[9] Johnson N P, McComb D W, Richel A, Treble B M and De la Rue R M 2001 Synthetic Met. 116469.

[10] Love J C, Gates B D, Wolfe D B, Paul K E and Whitesides G M 2002 Nano. Lett. 2891.

[11] Charnay C, Lee A, Man S Q, Moran C E, Radloff C, Bradley R K and Halas N J 2003 J. Phys. Chem. B 1077327.

[12] Lu Y, Liu G L, Mehia Y X and Lee L P 2005 Nano Lett. 5119.

[13] Liu J, Maaroof A I, Wieczorek L and Cortie M B 2005 Adv. Mater. 171276.

[14] Takei H, Himmelhaus M and Okamoto T 2002 Opt. Lett. 27342.

[15] Hulteen J C and Vanduyne R P 1995 J. Vac. Sci. Technol. A 131553.

[16] Yan F and Goedel W A 2004 Nano Lett. 41193.

[17] Han S B, Shi X Y and Zhou F M 2002 Nano Lett. 2435.

[18] Huang Z P, Carnahan D L, Rybczynski J, Giersig M, Sennett M, Wang D Z, Wen J G, Kempa K and Ren Z F 2003 Appl. Phys. Lett. 82460.

[19] Bohren C F and Huffman D R 1998 Absorption and Scattering of Light by Small Particles, Wiley, New York.

[20] Kaltchev M and Tysoe W T 1999 Surf. Sci. 43029.

[21] Stockli T, Bonard J M, Stadelmann P A and Chatelain A 1997 Z Phys. D Atom Mol. Cl. 40425.

[22] Neeves A E and Birnboim M H 1989 J. Opt. Soc. Am. B: Opt. Phys. 6787. 


\section{Aluminium 'nano-caps'}

[23] Draine B T and Flatau P J 1994 J. Opt. Soc. Am. A-Optics Image Sci. and Vision 111491.

[24] Foley J D, Dam A V, Feiner S K and Hughes J F 1990 Computer Graphics Second Edition AddisonWesley, Reading Massachusetts.

[25] Hoffmann A, Lenkefi Z and Szentirmay Z 1998 J. Phys-Condens. Mat. 105503.

[26] Quinten M and Kreibig U 1986 Surf. Sci. 172557.

[27] Kreibig U and Vollmer M 1995 Optical Properties of Metal Clusters Springer, New York.

[28] Prodan E, Radloff C, Halas N J and Nordlander P 2003 Science 302419.

[29] Wang H, Goodrich G P, Tam F, Oubre C, Nordlander P and Halas N J 2005 J. Phys. Chem B 109 11083. 